\title{
Article \\ Numerical Solutions of the Hattendorff Differential Equation for Multi-state Markov Insurance Models
}

\author{
Nathan Ritchey ${ }^{1, *,+}$ and Rajeev Rajaram ${ }^{2,+}$ \\ 1 Department of Mathematical Sciences Kent State University, Kent, OH 44242, USA, nritche2@kent.edu \\ 2 Department of Mathematical Sciences Kent State University, Kent, OH 44242, USA rrajaram@kent.edu \\ + These authors contributed equally to this work.
}

Abstract: We provide methodology and numerical results for the Hattendorf differential equation for the continuous time evolution of the variance of $L_{t}^{(j)}$, the loss at time $t$ random variable for a multi-state process, given that the state at time $t$ is $j$.

Keywords: Policy value; variance; standard deviation; numerical computing; Matlab; multistate model; thiele's differential equation; Hattendorff differential equation

\section{Introduction}

Markov State Space models are used to model the transition of states with probabilities assigned for transitions under the Markov assumption. They are widely used to model the evolution of policy values (mean) of an insurance product [1]. However, an adaptation to computing the evolution of variances of losses in each state is new and is the main focus of this paper.

The Hattendorff theorem, which was first considered in [2], proves that the yearly losses in a Markovian insurance policy are uncorrelated, and hence the variance of the total loss is equal to the sum of the variances of individual losses. In recent years, this topic has been re-visited using techniques of stochastic processes and martingales [3,4], [5], [6], [7]. Explicit formulas for the variance of the loss at time $t$ random variable ${ }^{2} \sigma_{t}^{(j)}$, given that the state at time $t$ is $j$, were derived in [8]. Differential equations for higher order moments and variances were derived in a more abstract form in [9] and [10]. A more recent paper in [11] derived a matrix representation for all higher-order moments for the loss at time $t$ of the random variable $L_{t}$.

In a previous paper, [12], we presented an explicit derivation of a differential equation (which we named as the Hattendorff Differential Equation) that describes the evolution of the variance of the loss at time $t$ in state $j$ random variable, denoted by $L_{t}^{j}$ (variance denoted by ${ }^{2} \sigma_{t}^{(j)}$ ) in the continuous time case. Along the way, we derived an explicit recursion formula for the discrete time case for annual and $h$-yearly cash flows. In this paper, we exploit the derived representation of the continuous time differential equation using MATLAB and compute ${ }^{2} \sigma_{t}^{(j)}$ as the solution of a backwards in time differential equation. We demonstrate this process by solving examples of several instances of a multi-state model in which the practitioner can use as an example for their own specific multi-state model.

The main goals of this paper are as follows:

1. Employ the differential equation that was derived in the the previous paper that governs the continuous time evolution of the variance of the loss at time $t$ random variable given that the state at time $t$ is $j\left(\right.$ denoted by ${ }^{2} \sigma_{t}^{(j)}$ ), for a general setting of benefits and premiums in the setting of a multistate Markov insurance model, to numerically solve specific examples.

2. Demonstrate how Matlab can be used to solve examples when they arise for the end user. 
3. Provide tips and advice on solving the Hattendorf differential equation.

As promised in the previous paper, [12], this paper provides the actual computation/approximation of ${ }^{2} \sigma_{t}^{(j)}$ for all time $t$ as a matter of solving a system of coupled differential equations. To our knowledge, this is the first time numerical solutions of this general insurance model for computation of the variance of loss ${ }^{2} \sigma_{t}^{(j)}$ have been considered.

The paper is organized as follows. In Section 2, we include our methods by stating notation, assumptions, an important theorems from the previous paper. We also present terminal conditions and an introduction to numerical computations in Section 3. In Section 4, we present and solve three particular models that can serve as a roadmap on how Matlab can be used to solve the differential equation describing the evolution of ${ }^{2} \sigma_{t}^{(j)}$ by using the $h$-yearly recursion stated in Section 2 . Finally, we conclude with a discussion in Section 5.

\section{Methods}

In line with [13]-Chapter 8 , we consider a multi-state model with $m+1$ states labeled as $0,1, \ldots, m$ with instantaneous transitions possible between pairs of states. We use $Y(t)$ to represent the state at time instant $t .\{Y(t)\}_{t \geq 0}$ is a stochastic process for $t \geq 0$ (continuous or discrete time depending on when we choose to make observations), with $Y(t)=i$ to mean that the individual is in state $i$ at age $x+t$ (initial age is taken as $x$ ).

We introduce the following notation for states $i$ and $j$ and ages $x \geq 0$ as in [13]Chapter 8:

\section{Notation}

1. $V_{t}^{(j)}=E\left[L_{t}^{(j)}\right]$

2. ${ }^{2} \sigma_{t}^{(j)}=\operatorname{Var}\left[L_{t}^{(j)}\right]$

3. $W_{t+h}^{(j k)}=V_{t+h}^{(k)}+h B_{t+h}^{(k)}-S_{t+h}^{(j k)}$ and $W_{t}^{(j k)}=\lim _{h \rightarrow 0} W_{t+h}^{(j k)}=V_{t}^{(k)}-S_{t}^{(j k)}$.

4. Represent the variance vector as

$$
\overrightarrow{{ }^{2} \sigma_{t}}=\operatorname{Var}\left(L_{t}\right)=\left[2 \sigma_{t}^{(0)},{ }^{2} \sigma_{t}^{(1)}, \ldots,{ }^{2} \sigma_{t}^{(m)}\right]^{T}
$$

5. Define the vector of variance of losses as

$$
{ }^{2} \sigma_{t}^{d}=\operatorname{Var}\left(L_{t}^{d}\right)=\left[{ }^{2} \sigma_{t}^{(0), d},{ }^{2} \sigma_{t}^{(1), d}, \ldots,{ }^{2} \sigma_{t}^{(m), d}\right]^{T} .
$$

6. Define the $j$-th element of the vector $\overrightarrow{\mu_{x+t}^{d}}$ as

$$
\left\{\mu_{x+t}^{\vec{d}}\right\}_{j}=\sum_{l \neq j} \mu_{x+t}^{j l}\left(W_{t}^{(j j)}-W_{t}^{(j l)}\right)^{2} .
$$

\section{Assumptions}

1. (Markovian assumption) For any states $i, j$ and times $t$ and $t+s$, the conditional probability $\operatorname{Pr}[Y(t+s)=j \mid Y(t)=i]$ is well defined and is independent of the knowledge of the process before time $t$.

2. The probability of two or more transitions in a time interval $h$ is $o(h)$.

3. For all states $i$ and $j$ and all ages $x \geq 0$, we assume that ${ }_{t} p_{x}^{i j}$ is a differentiable function of $t$.

4. For simplicity, we assume that there are no expenses although expenses can be easily incorporated into the formulation.

5. For simplicity, we assume a constant interest rate $i$ although a variable interest rate can be easily incorporated into the formulation. 
In this section, we list the main theorem that is proven in our previous paper, [12], which provides a continuous time differential equation satisfied by ${ }^{2} \sigma_{t}^{(j)}$ that mirrors the traditional Hattendorff recursion for the alive-dead model, but includes the option for multiple states.

Theorem 1. Assume that the above Notation is used and the Assumptions are true. Then ${ }^{2} \sigma_{t}^{(j)}$ satisfies the following differential equation:

$$
\begin{array}{r}
\frac{d}{d t}{ }^{2} \sigma_{t}^{(j)}=\left(2 \delta+\sum_{k \neq j} \mu_{x+t}^{j k}\right) \cdot{ }^{2} \sigma_{t}^{(j)}-\sum_{k \neq j} \mu_{x+t}^{j k} \cdot{ }^{2} \sigma_{t}^{(k)} \\
-\sum_{l \neq j} \mu_{x+t}^{j l}\left(W_{t}^{(j j)}-W_{t}^{(j l)}\right)^{2} .
\end{array}
$$

Note that we can use matrices to represent the theorem above.

\section{Terminal conditions}

In this section, we introduce terminal conditions and outline the steps involved to compute $\overrightarrow{2} \sigma_{t}$ numerically.

\section{1. $N$ year term or endowment insurance}

We consider end conditions for an $N$ year term (no survival benefit) or endowment insurance (survival benefit). In this case, all benefits at the end of the term are certain and hence the variance equals 0 . That is, ${ }^{2} \sigma_{N}=\overrightarrow{0}$.

\subsection{Numerical Solutions}

We use Matlab to solve specific instances of this model. Of course, any of the well-known solvers can be used. It is important to note that solving instances of this problem requires solving either two systems of differential equations, first solving one then inputting results into the other, or solving one larger coupled system. Because of the size of most applicable problems, it is quite efficient to simply solve the larger system of differential equations. Also, this system of differential equations has terminal conditions, as opposed to initial conditions. Most commercial codes require inital conditions. For our model, we have a backwards in time system. Thus, we can either develop a program that manages this, or use a change of variable technique to transform the terminal conditions to initial conditions. Since the change of variable is quite straight forward, we chose this route so that the most basic solver could be used.

To transform our problem, let $s=t_{0}-t$ where $t_{0}$ is the length of a policy. We also need to use the Chain Rule for this transformation and note that $\mathrm{dt} / \mathrm{ds}=-1$.

We used Matlab to perform the calculations required to find numerical solutions to three instances of the multi-state model. In each case, it took Matlab only a fraction of a second to find a solution to our problems.

Matlab can be organized in terms of subroutines or ".m" files. For the examples contained in this paper, we used two files, one to define the tansformed system of differential equations and the other, which we called the "driver." The driver was used to call the solver, input the particular system to solve, transform the solution back from variable $s$ to $t$, and then to plot the figures and generate paricular values of system. Matlab has several solvers that are used to solve systems of differential equations. We chose "ode45",' a built-in and popular differential equations solver to do the work. These files can be supplied upon request. 


\section{Results - Computational Examples}

In this section, we present four examples, each with an increasing number of states. We obtained our results using Matlab, a commercially available numerical computations product, to numerically approximate the solutions to these problems.

\subsection{Example 1: Continuous time classic insurance model}

For the first model, a two state model (alive and dead), we use an example that was found as Example 7.13, [1]. In this example, a twenty-year endowment insurance issued to a life aged 30 . The insured value is $\$ 100,000$ and payable immediately on death, or on survival to the end of the term, whichever occurs sooner. Premiums are payable continuously at a constant rate of $\$ 2500$ per year throughout the term of the policy. The policy value basis uses a constant force of interest, $\delta=0.04$ per year, and makes no allowance for expenses. In this example, we calculate the value $V_{10}$ and its standard deviation $\sqrt{{ }^{2} \sigma_{10}}$ using the general model that was presented in [12].

Using this information $n=20, P=2500$, age $=30$, and $S=100$, 000 we calculate $\mu_{50}=0.0015$. Thus, the problem is to solve the system of differential equations:

$$
\begin{aligned}
V_{t}^{\prime} & =.04115 V_{t}+2385 \\
\sigma_{t}^{\prime} & =.08115 \sigma_{t}-.00115\left(V_{t}-100000\right)^{2}
\end{aligned}
$$

Using Matlab, the solution is shown in Figure 1.

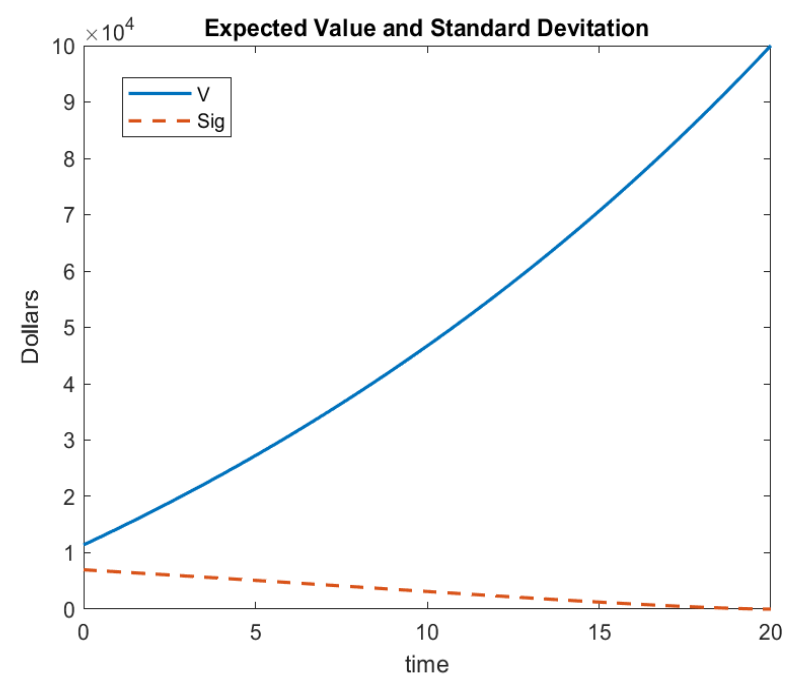

Figure 1

Also,

$$
V_{10} \approx 46,692 \text { and } \sqrt{{ }^{2} \sigma_{10}} \approx 3461 .
$$

Note that $V_{10}$ from this model agrees with that which is calculated in the textbook. 
4.2. Example 2: Continuous time disability income insurance model

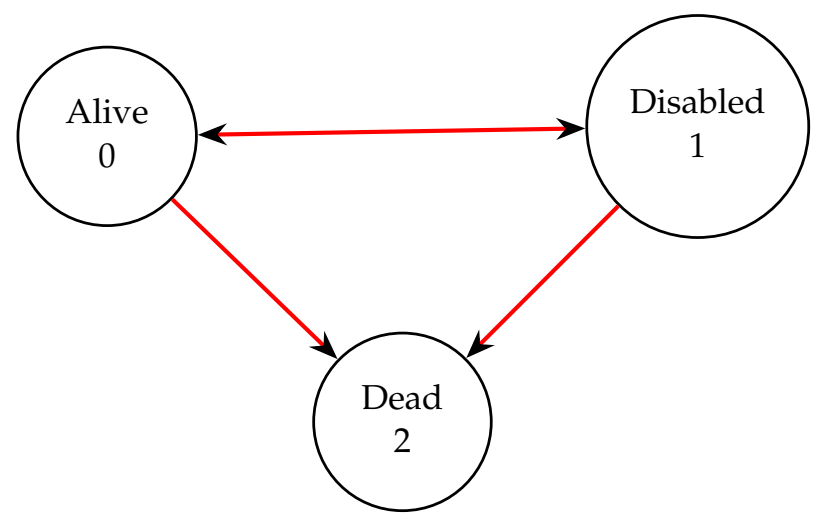

Figure 2

Consider a disability income insurance model with three states, alive, temporary disability, and dead, as shown in Figure 2. We also assume that the following transition rates apply to this example:

$$
\mu_{60+t}^{01}=0.05, \quad \mu_{60+t}^{02}=0.25 t, \quad \mu_{60+t}^{10}=0.25, \quad \mu_{60+t}^{12}=0.04 t
$$

The valuation force of interest is $5 \%$. You are also given:

$$
\bar{a}_{60}^{00}=5.1716, \quad \bar{a}_{60}^{01}=0.8430, \quad \bar{A}_{60}^{02}=0.6980, \quad \bar{a}_{60}^{11}=4.8201, \quad \bar{A}_{60}^{12}=0.7350,
$$

$\bar{a}_{70}^{00}=2.4769, \quad \bar{a}_{70}^{01}=0.2012, \quad \bar{A}_{70}^{02}=0.8659, \quad \bar{a}_{70}^{10}=0.1051, \quad \bar{a}_{70}^{11}=1.8528, \quad \bar{A}_{70}^{12}=0.9017$,

and

$$
{ }_{10} p_{60}^{00}=0.18314,{ }_{10} p_{60}^{01}=0.06181 \text {. }
$$

This 10-year health insurance example also has the following assurances.

- $\quad$ The product is issued to lives aged 60 in the healthy state.

- $\quad$ The product pays a death benefit of $\$ 5000$ at the moment of death.

- $\quad$ The product pays a continuous disability benefit at a rate of $\$ 750$ per year while the insured is temporarily disabled.

- $\quad$ Net premiums are payable continuously while the insured is healthy.

- $\quad$ The product pays an endowment of \$1000 if the person lives beyond 10 years.

The net premium can be computed using the Equivalence Premium Principle that is assumed to be at a rate of $P=\$ 695.64$.

Using this information, Thiele's differential equation, can be written as

$$
\frac{d}{d t}\left[\begin{array}{l}
V_{t}^{(0)} \\
V_{t}^{(1)}
\end{array}\right]=\left[\begin{array}{cc}
0.1+0.025 t & -0.05 \\
-0.025 & 0.075+0.04 t
\end{array}\right]\left[\begin{array}{c}
V_{t}^{(0)} \\
V_{t}^{(1)}
\end{array}\right]+\left[\begin{array}{c}
-125 t \\
695.64-(750+200 t)
\end{array}\right]
$$

Note that $V_{t}^{(2)}=0$ for all $t \geq 0$.

$$
\text { Terminal Conditions: }\left\{\begin{array}{l}
V_{10}^{(0)}=1000 \\
V_{10}^{(1)}=0
\end{array} \quad\right. \text { (Endowment) }
$$

Now let's consider Hattendorff's differential equation.

Recall that $\delta=0.05$ so $2 \delta=0.1$. 
Now,

$$
\begin{aligned}
\frac{d}{d t}\left[\begin{array}{l}
2 \sigma_{t}^{(0)} \\
{ }^{2} \sigma_{t}^{(1)} \\
{ }^{2} \sigma_{t}^{(2)}
\end{array}\right]= & {\left[\begin{array}{ccc}
\left.2 \delta+\mu_{60+t}^{01}+\mu_{60+t}^{02}\right) & -\mu_{60+t}^{01} & -\mu_{60+t}^{02} \\
-\mu_{60+t}^{10} & \left(2 \delta+\mu_{60+t}^{10}+\mu_{60+t}^{12}\right) & -\mu_{60+t}^{12} \\
0 & 0 & 2 \delta
\end{array}\right]\left[\begin{array}{c}
{ }^{2} \sigma_{t}^{(0)} \\
2 \\
{ }^{2} \sigma_{t}^{(1)} \\
{ }^{2} \sigma_{t}^{(2)}
\end{array}\right] } \\
- & {\left[\begin{array}{c}
\sum_{l \neq 0} \mu_{60+t}^{0 l}\left(V_{t}^{(0)}-\left(V_{t}^{(l)}-S_{t}^{(0 l)}\right)\right)^{2} \\
\sum_{l \neq 1} \mu_{60+t}^{1 l}\left(V_{t}^{(1)}-\left(V_{t}^{(l)}-S_{t}^{(1 l)}\right)\right)^{2} \\
\sum_{l \neq 2} \mu_{60+t}^{2 l}\left(V_{t}^{(2)}-\left(V_{t}^{(l)}-S_{t}^{(2 l)}\right)\right)^{2}
\end{array}\right] . }
\end{aligned}
$$

Also,

$$
\begin{gathered}
S_{t}^{(01)}=0, S_{t}^{(02)}=5000, S_{t}^{(10)}=0, S_{t}^{(12)}=5000 \\
W_{t}^{j j}=V_{t}^{(j)}-S_{t}^{(j j)}=V_{t}^{(j)}, W_{t}^{00}=V_{t}^{(0)}
\end{gathered}
$$

and

$$
W_{t}^{(j l)}=V_{t}^{(l)}-S_{t}^{(j l)}
$$

Therefore,

$$
\begin{aligned}
\frac{d}{d t}\left[\begin{array}{l}
2 \sigma_{t}^{(0)} \\
2 \sigma_{t}^{(1)} \\
2 \sigma_{t}^{(2)}
\end{array}\right]= & {\left[\begin{array}{ccc}
0.15+0.025 t & -0.05 & -0.025 t \\
-0.025 & 0.125+0.04 t & -0.04 t \\
0 & 0 & 0.1
\end{array}\right]\left[\begin{array}{c}
2 \sigma_{t}^{(0)} \\
2 \sigma_{t}^{(1)} \\
2 \sigma_{t}^{(2)}
\end{array}\right] } \\
- & {\left[\begin{array}{l}
0.05\left(V_{t}^{(0)}-V_{t}^{(1)}\right)^{2}+0.025 t\left(5000+V_{t}^{(0)}-V_{t}^{(2)}\right)^{2} \\
0.025\left(V_{t}^{(1)}-V_{t}^{(0)}\right)^{2}+0.04 t\left(5000+V_{t}^{(1)}-V_{t}^{(2)}\right)^{2} \\
0
\end{array}\right] . }
\end{aligned}
$$

Terminal Conditions are as follows:

$$
{ }^{2} \sigma_{10}^{(0)}={ }^{2} \sigma_{10}^{(1)}={ }^{2} \sigma_{10}^{(2)}=0 .
$$

These two systems of differential equations are coupled and they can be solved together. That is, we can combine the systems to obtain

$$
\begin{aligned}
& \frac{d}{d t}\left[\begin{array}{l}
V_{t}^{(0)} \\
V_{t}^{(1)} \\
2 \\
2 \sigma_{t}^{(0)} \\
2 \sigma_{t}^{(1)} \\
2 \sigma_{t}^{(2)}
\end{array}\right]=\left[\begin{array}{ccccc}
0.1+0.025 t & -0.05 & 0 & 0 & 0 \\
-0.025 & 0.075+0.04 t & 0 & 0 & 0 \\
0 & 0 & 0.15+0.025 t & -0.05 & -0.025 t \\
0 & 0 & -0.025 & 0.125+0.04 t & -0.04 t \\
0 & 0 & 0 & 0 & 0.1
\end{array}\right]\left[\begin{array}{c}
V_{t}^{(0)} \\
V_{t}^{(1)} \\
2 \sigma_{t}^{(0)} \\
2 \sigma_{t}^{(1)} \\
2 \sigma_{t}^{(2)}
\end{array}\right] \\
&- {\left[\begin{array}{l}
-125 t \\
-54.36-200 t) \\
0.05\left(V_{t}^{(0)}-V_{t}^{(1)}\right)^{2}+0.025 t\left(5000+V_{t}^{(0)}-V_{t}^{(2)}\right)^{2} \\
0.025\left(V_{t}^{(1)}-V_{t}^{(0)}\right)^{2}+0.04 t\left(5000+V_{t}^{(1)}-V_{t}^{(2)}\right)^{2} \\
0
\end{array}\right.}
\end{aligned}
$$

Notice that for these models, we have terminal conditions and not initial conditions. The differential equation solvers that are included with Matlab require initial conditions. Thus, we can simply use the substitution $t=10-s$ and the Chain Rule to rewrite the system so that the terminal conditions become initial conditions. Using the Matlab's 
'ode45' solver we have the following solutions for this system of differential equations. An identical solution is obtained when the example is presented to Matlab in matrix form. Note that one must rewrite the solution back to the variable $t$, once a solution has been obtained. Figure 3 represents the expected values and Figure 4 represents the standard deviations (square root of the variances) for this example.

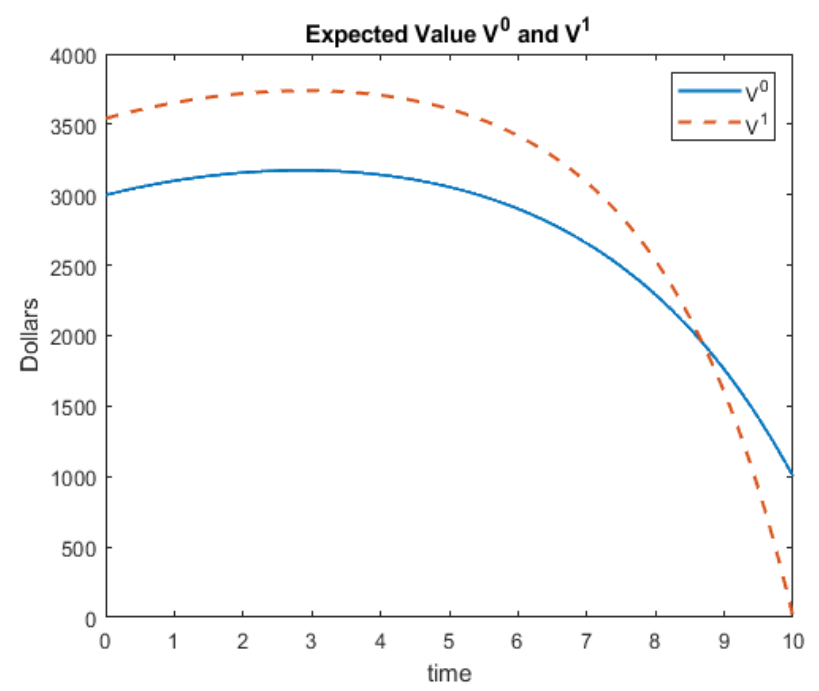

Figure 3

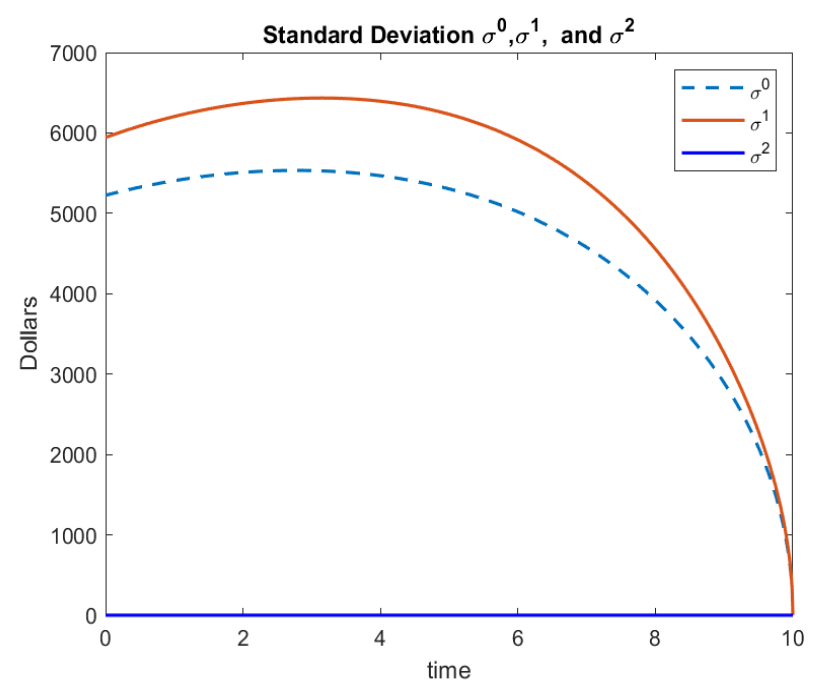

Figure 4

We end the example by stating the following:

1. We have used Matlab to solve this system of differential equations and acquired both $V_{t} i_{t}$ and ${ }^{2} \sigma_{t}^{(j)}$ in the process.

2. This step was easily accomplished using MATLAB. 


\subsection{Example 3}

We now consider a six state insurance model. Consider a disability income insurance model with six states: well (State 0$)$, temporary state of disability (State 1), temporary state of disability 2 (State 2), temporary state of disability 3 (State 3), temporary state of disability 4 (State 4), and dead (State 5), as indicated in Figure 5. We also assume that the following transition rates apply to this example:

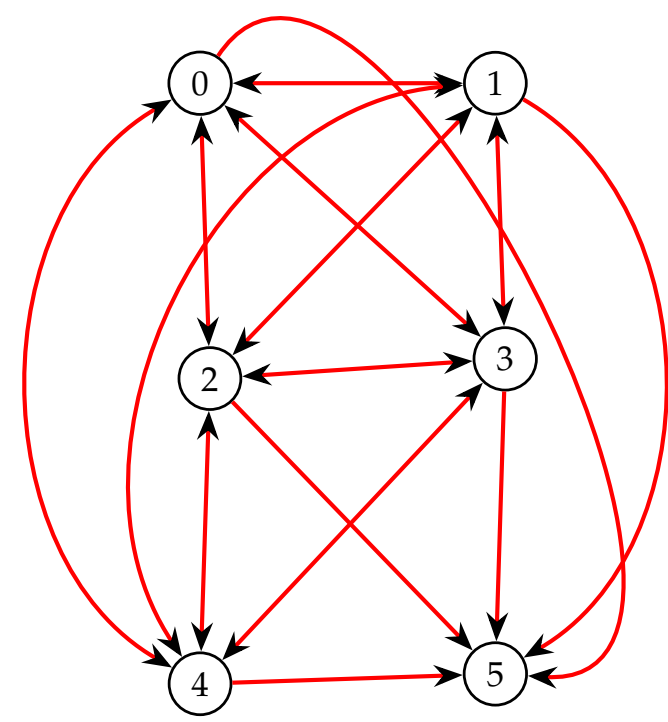

Figure 5

$$
\begin{aligned}
& \mu_{60+t}^{01}=0.01 t, \mu_{60+t}^{02}=0.015, \mu_{60+t}^{03}=0.01, \mu_{60+t}^{04}=0.01 \mu_{60+t}^{05}=0.018 \\
& \mu_{60+t}^{10}=0.06, \mu_{60+t}^{12}=0.01, \mu_{60+t}^{13}=0.020, \mu_{60+t}^{14}=0.01 t \mu_{60+t}^{15}=0.02 t \\
& \mu_{60+t}^{20}=0.055, \mu_{60+t}^{21}=0.15, \mu_{60+t}^{23}=0.025, \mu_{60+t}^{24 t}=0.027, \mu_{60+t}^{15}=0.035, \\
& \mu_{60+t}^{30}=0.05, \mu_{60+t}^{31}=0.045, \mu_{60+t}^{32}=0.015, \mu_{60+t}^{34}=0.018 t, \mu_{60+t}^{35}=0.038, \\
& \mu_{60+t}^{40}=0.04, \mu_{60+t}^{41}=0.045, \mu_{60+t}^{42}=0.025, \mu_{60+t}^{43}=0.028 t, \mu_{60+t}^{45}=0.18 t,
\end{aligned}
$$

The valuation force of interest is $5 \%$.

A 10-year health insurance product has the following features:

- $\quad$ The product is issued to lives aged 60 in the healthy state.

- $\quad$ The product pays a death benefit of $\$ 50,000$ at the moment of death.

- $\quad$ The product pays a continuous disability benefit at a rate of $\$ 2500$ per year while the insured is temporarily disabled.

- $\quad$ Net premiums are payable continuously while the insured is healthy.

- $\quad$ The product pays an endowment of $\$ 5000$ if the person lives beyond 10 years. 
You are also given enough information to use the Equivalence Premium Principle to calculate for this group to be offerred at a rate of $P=999.99$.

From this information, we have

$$
\begin{aligned}
& S_{t}^{(01)}=0, S_{t}^{(02)}=0, S_{t}^{(03)}=0, S_{t}^{(04)}=0, S_{t}^{(05)}=50,000 \\
& S_{t}^{(10)}=0, S_{t}^{(12)}=0, S_{t}^{(13)}=0, S_{t}^{(14)}=0, S_{t}^{(15)}=50,000 \\
& S_{t}^{(20)}=0, S_{t}^{(21)}=0, S_{t}^{(23)}=0, S_{t}^{(24)}=0, S_{t}^{(25)}=50,000 \\
& S_{t}^{(30)}=0, S_{t}^{(31)}=0, S_{t}^{(32)}=0, S_{t}^{(34)}=0, S_{t}^{(35)}=50,000 \\
& S_{t}^{(40)}=0, S_{t}^{(41)}=0, S_{t}^{(42)}=0, S_{t}^{(43)}=0, S_{t}^{(45)}=50,000 .
\end{aligned}
$$

By Thiele's differential equation, we have the following:

$$
\begin{aligned}
\frac{d}{d t}\left(V_{t}^{(0)}\right)= & 0.05 V_{t}^{(0)}+999.99-\left\{0.01 t\left(V_{t}^{(1)}-V_{t}^{(0)}\right)+0.015\left(V_{t}^{(2)}-V_{t}^{(0)}\right)\right. \\
& \left.+0.01\left(V_{t}^{(3)}-V_{t}^{(0)}\right)+0.01\left(V_{t}^{(4)}-V_{t}^{(0)}\right)+0.018\left(50,000+V_{t}^{(5)}-V_{t}^{(0)}\right)\right\} \\
= & V_{t}^{(0)}(0.05+0.01 t+0.015+0.01+0.01+0.018)+999.99-0.01 t V_{t}^{(1)}-0.015 V_{t}^{(2)} \\
& \quad-0.01 V_{t}^{(3)}-0.01 V_{t}^{(4)}-0.018(50,000) \\
= & V_{t}^{(0)}(0.103+0.01 t)-0.01 t V_{t}^{(1)}-0.015 V_{t}^{(2)}-0.01 V_{t}^{(3)}-0.01 V_{t}^{(4)}+99.99 .
\end{aligned}
$$

Note that $V_{t}^{(5)}=0$ for all $t \geq 0$.

In a similar manner, we have the following:

$$
\begin{aligned}
\frac{d}{d t}\left(V_{t}^{(1)}\right) & =-0.06 V_{t}^{(0)}+(0.14+.03 t) V_{t}^{(1)}-0.01 V_{t}^{(2)}-0.02 V_{t}^{(3)}-0.01 t V_{t}^{(4)}+2500+100 t \\
\frac{d}{d t}\left(V_{t}^{(2)}\right) & =-0.055 V_{t}^{(0)}-0.15 V_{t}^{(1)}+0.342 V_{t}^{(2)}-0.25 V_{t}^{(3)}-0.27 V_{t}^{(4)}-4250 \\
\frac{d}{d t}\left(V_{t}^{(3)}\right) & =-0.05 V_{t}^{(0)}-0.045 t V_{t}^{(1)}-0.015 V_{t}^{(2)}+(0.198+0.018 t) V_{t}^{(3)}-0.018 t V_{t}^{(4)}-4400 \\
\frac{d}{d t}\left(V_{t}^{(4)}\right) & =-0.04 V_{t}^{(0)}-0.045 V_{t}^{(1)}-0.025 V_{t}^{(2)}-0.028 t V_{t}^{(3)}+(0.16+0.208 t) V_{t}^{(4)} \\
& -2500+9000 t .
\end{aligned}
$$


In matrix form we have

$$
\begin{aligned}
& \frac{d}{d t}\left[\begin{array}{l}
V_{t}^{(0)} \\
V_{t}^{(1)} \\
V_{t}^{(2)} \\
V_{t}^{(3)} \\
V_{t}^{(4)}
\end{array}\right]= {\left[\begin{array}{ccccc}
(0.103+0.01 t) & -0.01 t & -.015 & -0.01 & -0.01 \\
-.06 & 0.14+.03 t) & -0.01 & -0.02 & -.01 t \\
-0.055 & -0.15 & 0.342 & -0.25 & -0.27 \\
-0.05 & -0.045 t & -0.015 & (0.198+0.018 t) & 0.018 t \\
-0.04 & -0.045 & -0.025 & -0.028 t & (0.16+0.208 t)
\end{array}\right]\left[\begin{array}{l}
V_{t}^{(0)} \\
V_{t}^{(1)} \\
V_{t}^{(2)} \\
V_{t}^{(3)} \\
V_{t}^{(4)}
\end{array}\right] } \\
&=+\left[\begin{array}{l}
99 \\
2500+100 t \\
-4250 \\
-4400 \\
-2500+9000 t
\end{array}\right] \\
&=A\left[\begin{array}{c}
V_{t}^{(0)} \\
V_{t}^{(1)} \\
V_{t}^{(2)} \\
V_{t}^{(3)} \\
V_{t}^{(4)}
\end{array}\right]+B,
\end{aligned}
$$

where $A$ and $B$ are used to represent each matrix.

Since $V_{t}^{(5)}=0$ for all $t \geq 0$ we have no need for a differential equation for that particular equation.

$$
\text { Terminal Conditions: }\left\{\begin{array}{l}
V_{10}^{(0)}=5000 \quad \text { (Endowment) } \\
V_{10}^{(1)}=0 \\
V_{10}^{(2)}=0 \\
V_{10}^{(3)}=0 \\
V_{10}^{(4)}=0 \\
V_{10}^{(5)}=0
\end{array}\right.
$$

Now let's consider Hattendorff's differential equation in matrix form.

Recall that $\delta=0.05$ so $2 \delta=0.1$.

Now,

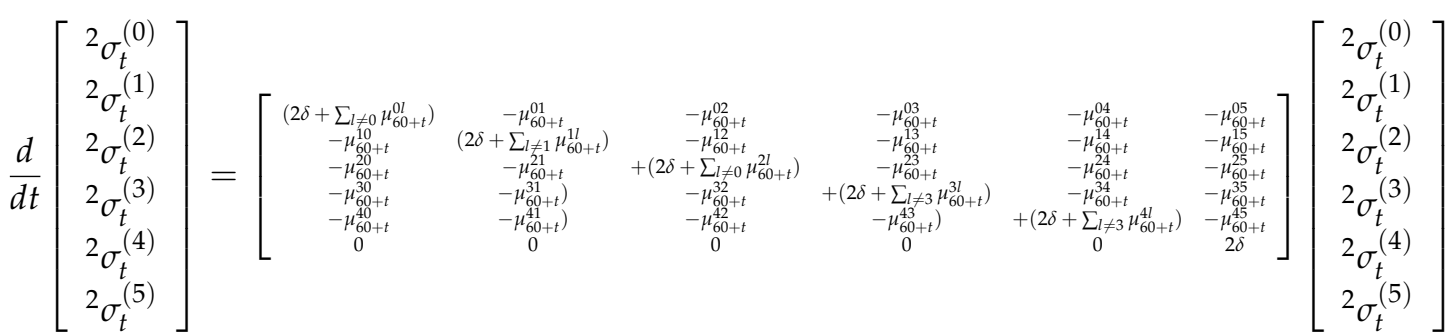

$$
+\left[\begin{array}{l}
\sum_{l \neq 0} \mu_{60+t}^{0 l}\left(V_{t}^{(0)}-\left(V_{t}^{(l)}-S_{t}^{(0 l)}\right)\right)^{2} \\
\sum_{l \neq 1} \mu_{60+t}^{1 l}\left(V_{t}^{(1)}-\left(V_{t}^{(l)}-S_{t}^{(1 l)}\right)\right)^{2} \\
\sum_{l \neq 2} \mu_{60+t}^{2 l}\left(V_{t}^{(2)}-\left(V_{t}^{(l)}-S_{t}^{(2 l)}\right)\right)^{2} \\
\sum_{l \neq 3} \mu_{60+t}^{0 l}\left(V_{t}^{(0)}-\left(V_{t}^{(l)}-S_{t}^{(0 l)}\right)\right)^{2} \\
\sum_{l \neq 4} \mu_{60+t}^{1 l}\left(V_{t}^{(1)}-\left(V_{t}^{(l)}-S_{t}^{(1 l)}\right)\right)^{2} \\
0
\end{array}\right] .
$$


Substituting in our known values, we have

$$
\begin{aligned}
& \frac{d}{d t}\left[\begin{array}{c}
2 \\
\sigma_{t}^{(0)} \\
2 \sigma_{t}^{(1)} \\
2 \sigma_{t}^{(2)} \\
2 \sigma_{t}^{(3)} \\
2 \sigma_{t}^{(4)} \\
2 \sigma_{t}^{(5)}
\end{array}\right]=\left[\begin{array}{cccccc}
(0.143+0.01 t) & -0.01 & -0.015 & -0.01 & -0.01 & -0.018 \\
-0.06 & 0.13+0.03 t) & -0.01 & -0.02 & -0.01 t & -0.02 t \\
-0.055 & -0.15 & +0.392 & -0.025 & -0.027 & -0.035 \\
-0.05 & -0.045 & -0.015 & +(0.248+0.18 t) & -0.018 t & -0.038 \\
-0.04 & -0.045 & -0.025 & -0.028 & +0.21+0.208 t) & -0.038 \\
0 & 0 & 0 & 0 & 0 & 0.1
\end{array}\right]\left[\begin{array}{c}
2 \sigma_{t}^{(0)} \\
2 \sigma_{t}^{(1)} \\
2 \sigma_{t}^{(2)} \\
2 \sigma_{t}^{(3)} \\
2 \sigma_{t}^{(4)} \\
2 \sigma_{t}^{(5)}
\end{array}\right] \\
& -\left[\begin{array}{l}
0.01 t\left(V_{t}^{0)}-V_{t}^{(1)}\right)^{2}+0.015\left(V_{t}^{0)}-V_{t}^{(2)}\right)^{2}+0.01\left(V_{t}^{0)}-V_{t}^{(3)}\right)^{2}+0.01\left(V_{t}^{0)}-V_{t}^{(4)}\right)^{2}+0.018\left(50000+V_{t}^{0)}\right)^{2} \\
0.06\left(V_{t}^{1)}-V_{t}^{(0)}\right)^{2}+0.01\left(V_{t}^{1)}-V_{t}^{(2)}\right)^{2}+0.02\left(V_{t}^{1)}-V_{t}^{(3)}\right)^{2}+0.01 t\left(V_{t}^{1)}-V_{t}^{(4)}\right)^{2}+0.02 t\left(50000+V_{t}^{1)}\right)^{2} \\
0.055\left(V_{t}^{2)}-V_{t}^{(0)}\right)^{2}+0.15\left(V_{t}^{2)}-V_{t}^{(1)}\right)^{2}+0.025\left(V_{t}^{2)}-V_{t}^{(3)}\right)^{2}+0.027\left(V_{t}^{3)}-V_{t}^{(4)}\right)^{2}+0.035\left(50000+V_{t}^{2)}\right)^{2} \\
0.05\left(V_{t}^{3)}-V_{t}^{(0)}\right)^{2}+0.045\left(V_{t}^{3)}-V_{t}^{(1)}\right)^{2}+0.015\left(V_{t}^{3)}-V_{t}^{(2)}\right)^{2}+0.018 t\left(V_{t}^{3)}-V_{t}^{(4)}\right)^{2}+0.038\left(50000+V_{t}^{3)}\right)^{2} \\
0.04\left(V_{t}^{4)}-V_{t}^{(0)}\right)^{2}+0.045\left(V_{t}^{4)}-V_{t}^{(1)}\right)^{2}+0.025\left(V_{t}^{4)}-V_{t}^{(2)}\right)^{2}+0.028 t\left(V_{t}^{4)}-V_{t}^{(3)}\right)^{2}+0.018 t\left(50000+V_{t}^{4)}\right)^{2} \\
0
\end{array}\right] \\
& -C\left[\begin{array}{l}
2 \sigma_{t}^{(0)} \\
2 \sigma_{t}^{(1)} \\
2 \sigma_{t}^{(2)} \\
2 \sigma_{t}^{(3)} \\
2 \sigma_{t}^{(4)} \\
2 \sigma_{t}^{(5)}
\end{array}\right]+D
\end{aligned}
$$

where $C$ and $D$ are the matrices above. Note that $D$ is in terms of the solution to the first system of equations.

Terminal Conditions are as follows:

$$
{ }^{2} \sigma_{10}^{(0)}={ }^{2} \sigma_{10}^{(1)}={ }^{2} \sigma_{10}^{(2)}={ }^{2} \sigma_{10}^{(3)}={ }^{2} \sigma_{10}^{(4)}={ }^{2} \sigma_{10}^{(5)}=0 .
$$

These two systems of differential equations are coupled and they can be solved togther. That is, we can combine the systems to obtain

$$
\frac{d}{d t}\left[\begin{array}{c}
V_{t}^{(0)} \\
V_{t}^{(1)} \\
V_{t}^{(2)} \\
V_{t}^{(3)} \\
V_{t}^{(4)} \\
2 \sigma_{t}^{(0)} \\
2 \sigma_{t}^{(1)} \\
2 \sigma_{t}^{(2)} \\
2 \sigma_{t}^{(3)} \\
2 \sigma_{t}^{(4)} \\
2 \sigma_{t}^{(5)}
\end{array}\right]=\left[\begin{array}{cc}
A & 05 \times 6 \\
06 \times 5 & C
\end{array}\right]\left[\begin{array}{c}
V_{t}^{(0)} \\
V_{t}^{(1)} \\
V_{t}^{(2)} \\
V_{t}^{(3)} \\
V_{t}^{(4)} \\
2 \sigma_{t}^{(0)} \\
2 \sigma_{t}^{(1)} \\
2 \sigma_{t}^{(2)} \\
2 \sigma_{t}^{(3)} \\
2 \sigma_{t}^{(4)} \\
2 \sigma_{t}^{(5)}
\end{array}\right]+\left[\begin{array}{c}
B \\
D
\end{array}\right]
$$

Notice that for these models, we have terminal conditions and not initial conditions. The differential equations solvers that are included with Matlab are designed to require initial conditions. Thus, we can simply use the substitution $t=10-s$ and the Chain Rule to rewrite the system so that the terminal conditions become initial conditions. Using the Matlab's 'ode45' solver we have the following solutions for this system of 
differential equations. An identical solution is obtained when the example is presented to Matlab in matrix form. Note that one must rewrite the solution back to the variable $t$, once a solution has been obtained. Figure 6 represents the expected values and Figure 7 represents the standard deviations (square root of the variances) for this example.

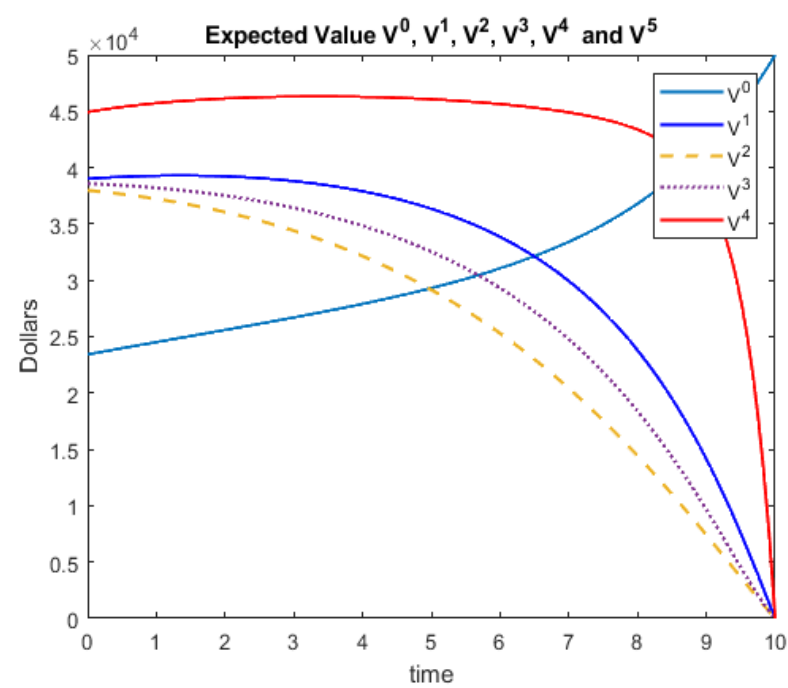

Figure 6

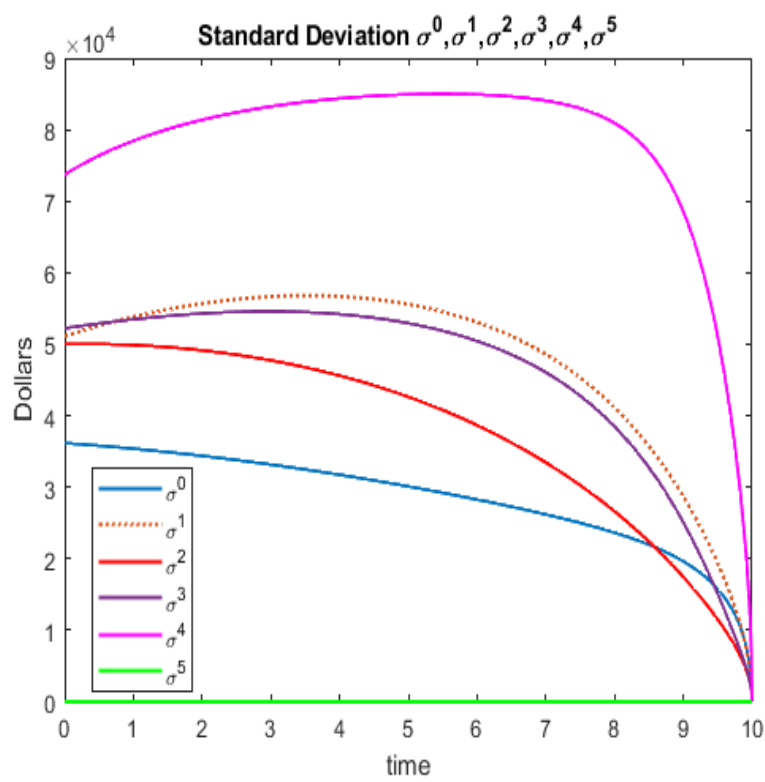

Figure 7

\section{Discussion}

In this paper, we have used the Hattendorff differential equation that we derived in [12], which describes the continuous time evolution of the variance of the loss at time $t$ random variable given that it is in state $j$ denoted by ${ }^{2} \sigma_{t}^{(j)}$ for continuous cash flows, along with terminal conditions. Matlab was used in three specific examples to numerically solve the Hattendorff differential equation for ${ }^{2} \sigma_{t}^{(j)}$. Furthermore, during the demonstrations, advice specific to setting up the Hattendorff differential equation for general multi-state models was given. 
We have demonstrated that solutions for various instances of the problem can be easily approximated using Matlab (or similar software). We have shown the adaptation and closed form expressions for the differential equation and recursion for three examples.

To our knowledge, this is the first time such expressions have been used to solve specific instances for the continuous time and discrete time evolution of ${ }^{2} \sigma_{t}^{(j)}$ for the multi-state case.

\section{References}

1. $\quad$ Bowers, N.L.; Gerber, H.U.; Hickman, J.C.; Jones, D.A.; Nesbitt, C.J. Actuarial Mathematics; The Society of Actuaries: Illinois, 1986.

2. Hattendorff, K. Das Risiko bei der Lebenversicherung. Masius Rundschau der Versi- 1868, 18, 169-183.

3. Gerber, H.U. An Introduction to Mathematical Risk Theory.; Huebner Foundation: Univ. of Pennsylvania, Philadelphia, 1979.

4. Gerber, H.U. Lebellsversicherungsmathematik; Springer-Verlag: Berlin, Heidelberg, New York, 1986.

5. Papatriandafylou, A.; Waters, H.R. Martingales in life insurance. Scand. Actuarial J. 1984, 1984, $210-230$.

6. Ramlau-Hansen, H. Hattendorff's theorem: a Markov chain and counting process approach. Scand. Actuarial J. 1988, 1987, 143156.

7. Norberg, R. Hattendorff's theorem and Thiele's differential equation generalized. Scand. Actuarial J. 1992, 1992, 1:2-14.

8. Wolthuis, H. Hattendorff's theorem for a continuous-time Markov model. Scand. Actuarial J. 1987, 1987, $157-175$.

9. Norberg, R. Differential Equations for moments of present values in life insurance. Insurance: Mathematics and Economics 1995, 17, 171-180.

10. Asmussen, S.; Steffensen, M. Risk and Insurance; Springer, 2020.

11. Bladt, M.; Asmussen, A.; Steffensen, S. Matrix representations of life insurance payments. European Actuarial J. $2020,10,29-67$.

12. Rajaram, R.; Ritchey, N. Hattendorff Differential Equation for Multi-State Markov Insurance Models. Risks 2021, 9, 169-187.

13. Dickson, C.M.D.; Hardy, M.R.; Waters, H.R. Actuarial Mathematics for Life Contingent Risks; Cambridge University Press, 2020. 